\title{
Serial Acquisition of DS-CDMA Signals in Multipath Fading Mobile Channels
}

\author{
Lie-Liang Yang, Member, IEEE, and Lajos Hanzo, Senior Member, IEEE
}

\begin{abstract}
This paper compares the mean acquisition time (MAT) performance of two serial search acquisition schemes over frequency-selective multipath Rayleigh fading channels. Both the conventional cell-by-cell detection and the novel joint two-cell detection are characterized. In contrast to the majority of published results considering only one correct timing state $\left(H_{1}\right.$ cell) in the uncertainty region of the pseudonoise (PN) sequence, this paper analyzes the MAT performance of the serial search acquisition schemes with multiple timing states in the uncertainty region. The MAT performance comparison between the conventional cell-by-cell detection and the joint two-cell detection shows that the latter can achieve an improved acquisition performance at almost the same complexity.
\end{abstract}

Index Terms-Acquisition, direct-sequence code-division multiple-access, mean acquisition time, multipath fading channel, pseudonoise.

\section{INTRODUCTION}

$\mathbf{P}$ SEUDONOISE (PN) code synchronization is the necessary first step in the receiver of direct-sequence code-division multiple-access (DS-CDMA) systems, since data demodulation becomes possible only after the code synchronization is performed. Code synchronization is usually achieved in two steps: 1) acquisition for coarse code alignment and 2) tracking for fine alignment [1]. Acquisition methods can be broadly classified as serial search methods [2]-[9] and parallel search methods [3], [10]-[14], which have been extensively treated in the literature. In a serial search scheme, each possible code phase of a given roster position in the uncertainty region is tested one at a time, while in a parallel search strategy, many, if not all, of the possible code phases are tested simultaneously. The focus of this paper is on serial search acquisition.

In a practical PN sequence acquisition system, usually there exist multiple in-phase states ( $H_{1}$ cells) in the uncertainty region, since the search step size is usually lower than the chip duration and the dispersive fading-induced delay spread is higher than the chip duration of the PN sequence. However, despite the existence of multiple $H_{1}$ cells in almost any practical PN code acquisition system, the majority of published results concerning the mean acquisition time (MAT) have been derived from the assumption that there is one and only one $H_{1}$ cell in the uncertainty region [1]-[7], [9]-[14]. The performance of the acquisi-

Manuscript received October 29, 1998; revised August 2, 2000. This work was supported by EPSRC, U.K.; the Commission of the European Communities, Brussels, Belgium; and the Royal Society of British.

The authors are with the Department of Electronics and Computer Science, University of Southampton, Southampton SO17 1BJ, U.K. (e-mail: 1ly@ecs.soton.ac.uk).

Publisher Item Identifier S 0018-9545(01)03219-4. tion system with multiple $H_{1}$ cells has received comparatively little attention in the literature.

Hence, our goal in this paper is to quantify the performance of serial search acquisition systems under the hypothesis that there are multiple $H_{1}$ cells in the uncertainty region of the PN sequence. Specifically, we assume that the search step size is $T_{c} / 2$, where $T_{c}$ is the chip duration. Hence, each resolvable path of the received signal contributes two $H_{1}$ cells. The acquisition process of the serial search mode under the multiple $H_{1}$-cell hypothesis can be described using the so-called equivalent circular state diagram [2], [4], [17], which will be detailed in the context of Fig. 2. By extending the result of [17] in this paper, a generalized equation is derived for computing the MAT of a serial search system under the multiple $H_{1}$-cell hypothesis. In our analysis, two detection schemes are considered. The first is the conventional cell-by-cell detection [5], in which each cell is tested independently; the second is the novel joint two-cell detection [15], [16], in which two adjacent cells are tested jointly. The MAT performance of the serial search scheme using both of the above detection approaches is investigated over a frequency-selective multipath Rayleigh fading medium. Note that the analysis presented is applicable to the mobile-to-base station link of a DS-CDMA system utilizing binary phase-shift keying (BPSK) spreading.

The remainder of this paper is organized as follows. In Section II, we describe the search and detection modes proposed. Then, the asymptotic MAT is derived in Section III. The system and channel models are given in Sections IV and V. In Section VI, the test statistics of the decision variables are analyzed, while in Section VII we derive the overall miss probability and the false alarm probability, which are defined in Section III. Numerical results are given in Section VIII. Our concluding remarks are given in Section IX. Let us now consider the search and detection modes concerned in this paper.

\section{Search And Detection Modes}

The block diagram of the serial search mode and the detection mode is shown in Fig. 1. The received signal is first down-converted into in-phase $(I)$ and quadrature $(Q)$ components. Two $I$ - $Q$ correlators perform correlation between the locally generated PN code $c\left(t-i T_{c} / 2\right)$ and the $I$ as well as the $Q$ baseband signals in Fig. 1. This product is integrated over the interval of $\tau_{D}=M T_{c} \mathrm{~s}$, where $\tau_{D}$ represents the integral dwell time and $M$ is an integer. The outputs of the $I$ - $Q$ correlators are then squared and summed to give an output variable, which is denoted by $V_{i}$ in Fig. 1.

In this paper, two detection modes having a similar implementation complexity will be characterized. The first is the con- 


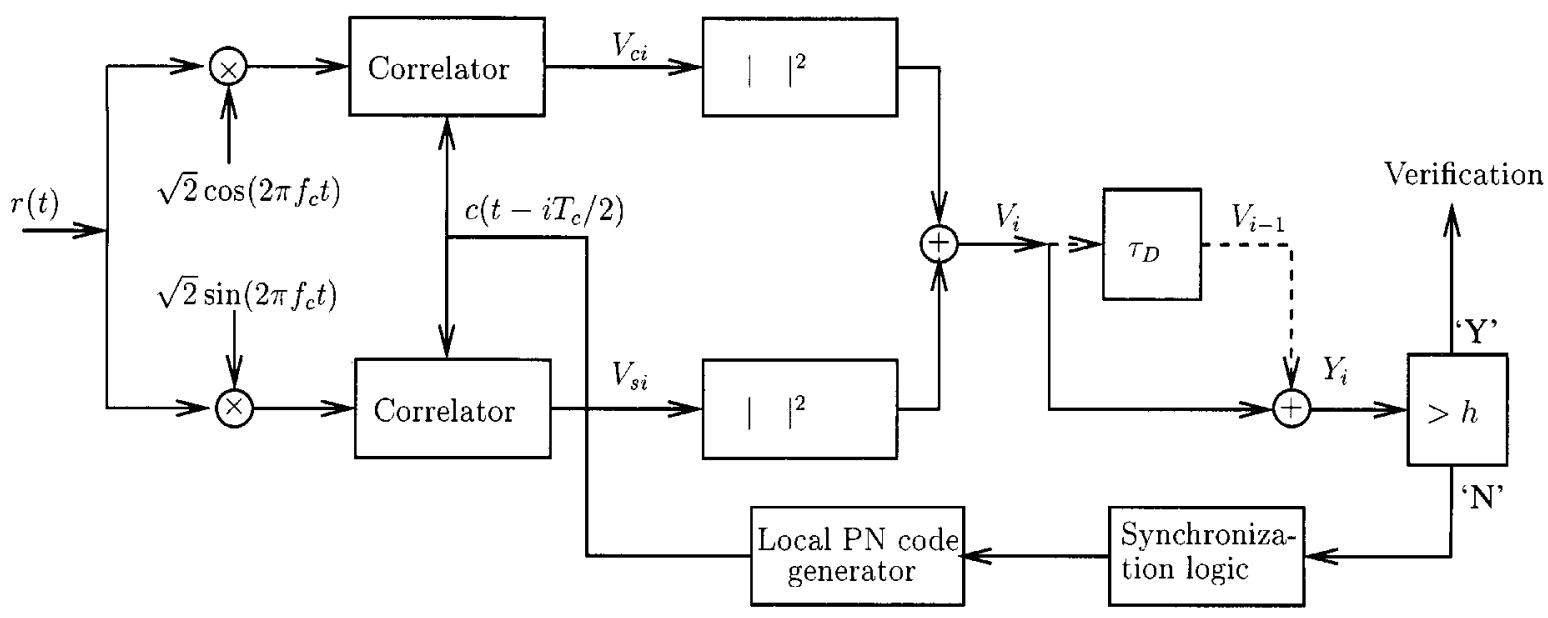

Fig. 1. Acquisition block diagram.

ventional cell-by-cell detection scheme [2], in which the cells in the uncertainty region are searched and tested cell by cell independently. The generation of the decision variable $Y_{i}$ is indicated by the continuous line in Fig. 1, which is expressed as $Y_{i}=$ $V_{i}$. The second detection mode is the so-called joint two-cell detection scheme [15], in which the search and detection block diagram includes both the continuous and dashed lines in Fig. 1. The statistics of the final decision variable are now given by the sum of two adjacent output variables, expressed as $Y_{i}=$ $V_{i-1}+V_{i}$. However, if the search mode detects a false alarm during the detection of $Y_{i-1}$, the term $V_{i-1}$ in the decision variable $Y_{i}$ is set to zero. Let $h$ in Fig. 1 be a decision threshold. Then, the search and detection mode can be described as follows. Whenever the decision variable $Y_{i}$ exceeds the threshold $h$, the system assumes that the corresponding delay of the locally generated PN sequence is the correct delay and proceeds to the verification mode. Otherwise, if $Y_{i}$ does not exceed $h$, the relative phase of the locally generated PN sequence is readjusted in order to update the decision variable $Y_{i}$, and the above process is repeated. Note that the verification mode is usually used to confirm a stronger decision concerning the correct delay assumed by the search mode [10], an issue not analyzed in this paper.

Since each cell is classified as one of the two states-correct phase (in-phase) of $H_{1}$ and incorrect phase of $H_{0}$, the first search mode with conventional cell-by-cell detection can be described as a two-state Markov process [5] owing to each decision variable being decided by one cell. The second search mode with joint two-cell detection can be described as a four-state random Markov process [15] owing to the final decision variable now being decided by two cells. Let us now derive a generalized equation for computing the MAT of the above schemes.

\section{Asymptotic MEAn ACQUisition TIME}

Holmes and Chen [5], as well as Polydoros and Weber [2], have given the equations for computing the MAT of a serial search code acquisition system in both exact and asymptotic forms under the single $H_{1}$-cell and double $H_{1}$-cell hypotheses in the uncertainty region. However, due to the finite search step

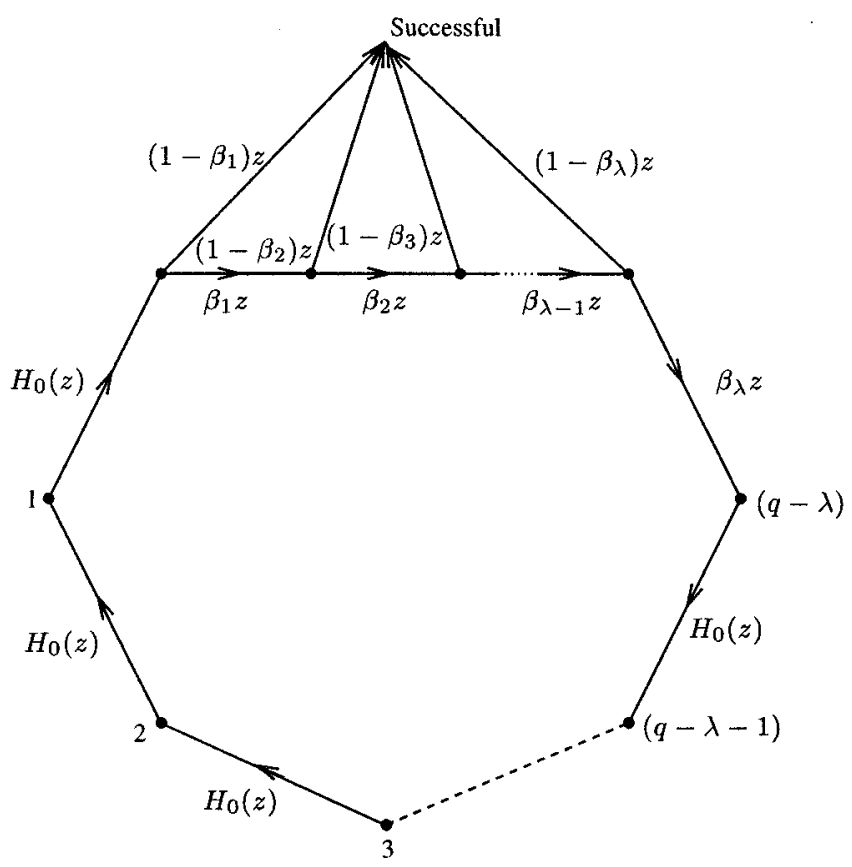

Fig. 2. Equivalent circular state diagram for a serial search code-acquisition system with multiple timing hypotheses.

size and due to receiving multiple delayed replicas of the transmitted PN sequences in a dispersive multipath fading environment, the actual situation is that there may exist more than two states, hence satisfying the multiple $H_{1}$-cell hypothesis. Therefore, in this section, we extend the result of [17] in order to derive a generalized asymptotic equation for the MAT, which includes the asymptotic expressions of [2], [5] as special cases.

The equivalent circular state diagram of [17] can be adapted for a serial search code acquisition system relying on the multiple $H_{1}$-cell hypothesis, which is redrawn in Fig. 2. $P_{D_{i}}$ is replaced by $1-\beta_{i}$ for notational convenience, where $P_{D_{i}}$ and $\beta_{i}$ represent the detection and miss probabilities of the $i$ th detection, leading to $\beta_{i}=1-P_{D_{i}}$. In Fig. 2, nodes represent states, labeled branches between two nodes represent state transitions, $z$ indicates the unit-delay operator, and the power of $z$ represents the time delay. Let us assume that there are a total of $q$ states, 
which includes $\lambda H_{1}$ cells and $(q-\lambda) H_{0}$ cells. Let $\alpha$ represent the false-alarm probability associated with an $H_{0}$ cell, i.e., the probability that an $H_{0}$ cell provides a correlator output in excess of $h$, hence resulting in an incorrect hypothesis and leading to an unsynchronized condition. Let $K \tau_{D}$ represent the "penalty time" associated with noticing that there is a false alarm and with reentering the search mode. Then, following the philosophy of [17] and assuming that we commence the search anywhere outside the correct states, the transfer function assuming equal prior probability of each state can be expressed as

$$
U(z)=\frac{H_{D}(z)}{(q-\lambda)\left[1-H_{M}(z) H_{0}^{q-\lambda}(z)\right]} \cdot \sum_{i=1}^{q-\lambda} H_{0}^{i}(z)
$$

where $H_{D}(z)$ and $H_{M}(z)$ include all paths leading to successful detection or miss, respectively. In (1), $H_{D}(z)$ and $H_{0}(z)$ can be expressed as

$$
\begin{aligned}
H_{D}(z) & =\sum_{j=1}^{\lambda}\left(1-\beta_{j}\right) z \prod_{i=1}^{j-1} \beta_{i} z, \quad \text { with } \prod_{i=1}^{0} \beta_{i} z=1 \\
H_{0}(z) & =(1-\alpha) z+\alpha z^{K+1} .
\end{aligned}
$$

Let $P_{M}(\lambda)=\prod_{i=1}^{\lambda} \beta_{i}$, which represents the overall miss probability of a search over the full uncertainty region. Then $H_{M}(z)$ in (1) is given by $H_{M}(z)=P_{M}(\lambda) z^{\lambda}$. The MAT can be expressed as (2) [5], shown at the bottom of the page.

In order to obtain the asymptotic form of the MAT for $q \gg 1$, we divide both sides of (2) by the total number of states $q$ and then compute its limit with respect to $q \rightarrow \infty$, yielding

$$
\lim _{q \rightarrow \infty} \frac{\bar{T}_{\mathrm{acq}}}{q}=\frac{\left[1+P_{M}(\lambda)\right](1+K \alpha)}{2\left[1-P_{M}(\lambda)\right]} \tau_{D}
$$

Consequently, if the total number of states $q$ is significantly higher than the number of $H_{1}$ cells, i.e., $q \gg \lambda$, then the MAT can be approximated by

$$
\bar{T}_{\mathrm{acq}} \approx \frac{\left[1+P_{M}(\lambda)\right](1+K \alpha)}{2\left[1-P_{M}(\lambda)\right]}\left(q \tau_{D}\right)
$$

Equation (4) is a generalized expression for computing the asymptotic MAT of the serial search acquisition system with single or multiple adjacent $H_{1}$ cells. For example, when there are two $H_{1}$ cells in the uncertainty region, then the overall miss probability of $P_{M}(2)=\beta_{1} \beta_{2}=\left(1-P_{D_{1}}\right)\left(1-P_{D_{2}}\right)$, where $P_{D_{1}}$ and $P_{D_{2}}$ represent the detection probabilities of the first and second $H_{1}$ cells, respectively. Upon substituting these conditions into (4), the asymptotic MAT for a serial search acquisition system having two $H_{1}$ cells can be expressed as

$$
\bar{T}_{\text {acq }} \approx \frac{\left[2-P_{D_{1}}-\left(1-P_{D_{1}}\right) P_{D_{2}}\right](1+K \alpha)}{2\left[P_{D_{1}}+\left(1-P_{D_{1}}\right) P_{D_{2}}\right]}\left(q \tau_{D}\right)
$$

which represents the asymptotic result given in [2, eq. (20)], when uniform distribution of the two $H_{1}$ cells over the entire uncertainty region is assumed.

\section{The Transmitted Signals}

The communication model under consideration consists of $U$ simultaneous transmitters, which includes $U-1$ data transmission users (who have finished acquisition) and one initial synchronization user [whose PN sequence is being acquired by the base station (BS)]. We assume that the first user is the initial synchronization user, whose performance is to be evaluated. Each user is assigned a unique CDMA sequence, which spreads the data sequence. Again, in this contribution, random sequences having a common chip rate of $1 / T_{c}$ are considered. The processing gain is given by $G=T / T_{c}$, and $1 / T$ is the information bit rate. Let $\left\{c_{j}^{(k)}\right\}$ denote a binary $\{+1,-1\}$ sequence, where $c_{j}^{(k)}$ assumes values of +1 and -1 with equal probability, and let $c_{k}(t)=\sum_{j=-\infty}^{\infty} c_{j}^{(k)} P_{T_{c}}\left(t-j T_{c}\right)$ denote the spreading sequence of the $k$ th user, where $P_{\tau}(t)=1$ for $0 \leq t \leq \tau$ and zero otherwise. The data waveform $b_{k}(t)=$ $\sum_{i=-\infty}^{\infty} b_{i}^{(k)} P_{T}(t-i T)$ consists of a sequence of mutually independent rectangular pulses of duration $T$ and of amplitudes +1 or -1 with equal probability.

The transmitted signal for the $k$ th user is expressed as

$$
s_{k_{i}}(t)=\sqrt{2 P_{k_{i}}} b_{k_{k}}(t) c_{k_{k}}(t) \cos \left(2 \pi f_{c} t+\varphi_{k_{i}}\right)
$$

where

$P_{k} \quad$ transmitted power of the $k$ th signal;

$f_{c} \quad$ common carrier frequency;

$\varphi_{k} \quad$ phase introduced by the $k$ th modulator, which is modeled as a random variable uniformly distributed over $[0,2 \pi)$.

In many DS-CDMA systems [2]-[10], the transmitter aids the initial synchronization by transmitting the phase-coded carrier signal without data modulation at the beginning of each transmission. Hence, in our analysis, we assume for simplicity that no data modulation is imposed on the initial synchronization signals.

\section{THE ChANNEL MODEL}

A widely accepted model for a frequency-selective multipath fading channel is a finite-length tapped delay line with a tap

$$
\begin{aligned}
\bar{T}_{\text {acq }} & =\left[\frac{d U(z)}{d z}\right]_{z=1} \tau_{D} \\
& =\frac{2 \cdot \sum_{j=0}^{\lambda-1} \beta_{j} \prod_{i=0}^{j-1} \beta_{i}+(q-\lambda+1)\left[1-P_{M}(\lambda)\right](1+K \alpha)+2(q-\lambda) P_{M}(\lambda)(1+K \alpha)}{2\left[1-P_{M}(\lambda)\right]} \tau_{D} .
\end{aligned}
$$




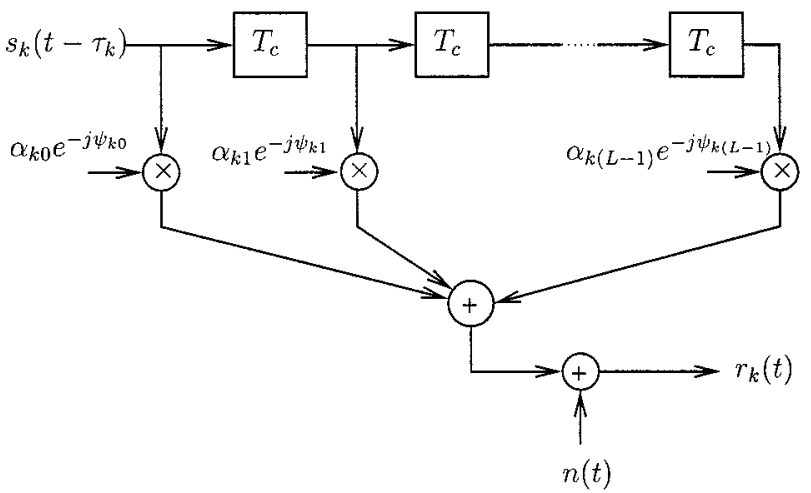

Fig. 3. Tapped delay line model of the frequency-selective channel.

spacing of one chip [18], as shown in Fig. 3, where the $L$ tap weights $\left\{\alpha_{k l}\right\}$ are assumed to be independent identically distributed (i.i.d.) Rayleigh random variables with a probability density function (pdf) given by [18]

$$
f_{\alpha_{k l}}(x)=\frac{2 x}{\Omega} \exp \left(-\frac{x^{2}}{\Omega}\right), \quad x \geq 0
$$

where $E\left[\alpha_{k l l}^{2}\right]=\Omega$, and the phases $\left\{\psi_{k l}\right\}$ are assumed i.i.d. random variables uniformly distributed in $[0,2 \pi)$. Furthermore, we assume that the fading amplitude is constant during an observation interval but changes from one interval to another independently. Then, when signals in the form of (6) are transmitted over the multipath Rayleigh fading channels of Fig. 3, the received signal can be expressed as

$$
\begin{aligned}
r(t)= & \sum_{l=0}^{L-1} \sqrt{2 P_{R}} \alpha_{1 l} c_{1}\left(t-\tau_{1}-l T_{c}\right) \cos \left(2 \pi f_{c} t+\theta_{1 l}\right) \\
& +\sum_{k=2}^{U} \sum_{l=0}^{L-1} \sqrt{2 P_{I}} \alpha_{k l} b_{k}\left(t-\tau_{k}-l T_{c}\right) \\
& \times c_{k}\left(t-\tau_{k}-l T_{c}\right) \cos \left(2 \pi f_{c} t+\theta_{k l}\right)+n(t)
\end{aligned}
$$

where $\tau_{k}$ is the relative time delay associated with an asynchronous transmission scheme; $\theta_{k l}=\varphi_{k}-\psi_{k l}-2 \pi f_{c}\left(\tau_{k}+l T_{c}\right)$ represents the i.i.d. random variables uniformly distributed in $[0,2 \pi)$; and $n(t)$ represents the additive white Gaussian noise (AWGN) with a double-sided power spectral density of $N_{0} / 2$. Note that since the $U-1$ interfering users are engaged in data transmission, we assume that their signals are ideally powercontrolled and the average received power of each interfering signal is expressed as $P_{I}$. However, for the initial synchronization user, it is unrealistic to invoke near-ideal power control before successful code acquisition. It can only rely on open-loop power control according to the estimation of the channel state. Hence, the average received power of the initial synchronization user at the BS is usually different from that of the data transmission users. This power is expressed in (8) as $P_{R}$.

\section{Test Statistics of the Decision Variable}

In this section, we derive the statistics of the decision variables for the serial search acquisition system using either conventional cell-by-cell detection or joint two-cell detection in the context of the noncoherent acquisition system over a frequency-selective multipath Rayleigh fading channel. In deriving the expressions of the statistics, we adopt the following assumptions.

1) The search step size is $T_{c} / 2$.

2) All samples are independent.

3) $M \gg 1$ such that the mean value of the correlation between the received and the locally generated PN sequences is zero when they are out of phase.

4) The self-interference of the desired signal due to multipath transmission and the multiple-access interference inflicted by the $U-1$ data transmission users can be modeled as additive Gaussian noise.

Referring to Fig. 1, the correlator output of the in-phase branch $V_{\text {ci }}$ gives the desired signal component, the multiple-access interference, and the Gaussian noise from $n(t)$ of (8). $V_{\mathrm{ci}}$ can be expressed as

$$
\begin{aligned}
V_{\mathbf{c i}} & =\int_{0}^{M T_{c}} r(t) c_{1}\left(t-i T_{c} / 2\right) \sqrt{2} \cos \left(2 \pi f_{c} t\right) d t \\
& =\sqrt{P_{R}} M T_{c}\left[\sum_{l=0}^{L-1} S_{I}(l)+\sum_{k=2}^{U} \sum_{l=0}^{L-1} M_{I}(k, l)+\eta_{I}\right]
\end{aligned}
$$

where $S_{I}(l)$ is the signal component due to the user-of-interest, which represents the desired signal component or the self-interference depending on whether the received and the locally generated PN sequences are in-phase or out of phase. $S_{I}(l)$ can be expressed as

$$
\begin{aligned}
& S_{I}(l) \\
& \quad=\frac{\alpha_{1 l} \cos \theta_{1 l}}{M T_{c}}\left[\tau_{1}^{\prime} R_{M}(i, N+1)+\left(T_{c}-\tau_{1}^{\prime}\right) R_{M}(i, N)\right]
\end{aligned}
$$

if $\tau_{1}+(l-i / 2) T_{c}=N T_{c}+\tau_{1}^{\prime}$, where $\tau_{1}$ was defined in (8) and $\tau_{1}^{\prime}$ is a random variable uniformly distributed in $\left[0, T_{c}\right]$. In (10), $R_{M}(i, N)$ represents the partial autocorrelation function defined as

$$
R_{M}(i, N)=\sum_{v=0}^{M-1} c_{v+i}^{(1)} \cdot c_{v+i+N}^{(1)} .
$$

Note that since a noncoherent acquisition system can acquire only one path of the multipath signal at any time, the other $L-1$ path signals of the reference user inflict self-interference to the in-phase path. Moreover, if the locally generated sequence is out of phase with any of the $L$ path signals of the reference user, all $L$ path signals of the user-of-interest constitute selfinterference.

Since random sequences are assigned for all users of the system, random data modulation does not change the statistics of the random sequences. Consequently, assuming that $\tau_{k}+(l-i / 2) T_{c}=N T_{c}+\tau_{k}^{\prime}$, where $\tau_{k}^{\prime}$ is a uniformly 
distributed random variable over $\left[0, T_{c}\right)$, the multiple-access interference term $M_{I}(k, l)$ can be expressed as

$$
\begin{aligned}
M_{I}(k, l)= & \frac{\sqrt{\rho} \alpha_{k l} \cos \theta_{k l}}{M T_{c}} \\
& \times\left[\tau_{k}^{\prime} R_{M}^{(k)}(i, N+1)+\left(T_{c}-\tau_{k}^{\prime}\right) R_{M}^{(k)}(i, N)\right]
\end{aligned}
$$

where $\rho=P_{I} / P_{R}$ and $R_{M}^{(k)}(i, N)$ represents the partial cross correlation between the sequence of the $k$ th user and the locally generated sequence, which is defined as

$$
R_{M}^{(k)}(i, N)=\sum_{v=0}^{M-1} c_{v+i}^{(1)} \cdot c_{v+i+N}^{(k)} \cdot
$$

Finally, the noise term $n(t)$ due to the presence of the AWGN can be expressed as

$$
\eta_{I}=\frac{1}{\sqrt{P_{R}} M T_{c}} \int_{0}^{M T_{c}} n(t) c_{1}\left(t-i T_{c} / 2\right) \sqrt{2} \cos \left(2 \pi f_{c} t\right) d t .
$$

The correlator output of the quadrature branch $V_{\mathrm{si}}$ can be obtained from the in-phase component if we replace $\cos ()$ by $-\sin ()$ in (10) and (12) and replace $\cos ()$ by $\sin ()$ in (14).

\section{A. Gaussian Approximation}

Recall that the spreading sequences of the system are modeled as random binary sequences of equiprobable $\pm 1 \mathrm{~s}$. Therefore, it can be shown that the terms of $V_{\mathrm{ci}}$ and $V_{\mathrm{si}}$ are asymptotically Gaussian due to the central limit theorem [19], [20] as the number of users increases. In our analysis, we employ the Gaussian approximation and model the self-interference due to multipath dispersion of the user-of-interest, the multiple-access interference due to the $U-1$ data transmission users, and the thermal noise as additive Gaussian noise. Consequently, in (10), if $N=0$ and $\tau_{1}^{\prime}$ is uniformly distributed in $\left[0, T_{c} / 2\right]$ as well as if $N=-1$ and $\tau_{1}^{\prime}$ is uniformly distributed in $\left[T_{c} / 2, T_{c}\right]$, two adjacent $H_{1}$ cells associated with one resolvable path are obtained. The mean value corresponding to these two $H_{1}$ cells in terms of $\tau_{1}^{\prime}$ can be expressed as [15]

$$
E\left[S_{I}(l)\right]_{N=0}=E\left[S_{I}(l)\right]_{N=-1}=\frac{3}{4} \alpha_{1 l} \cos \theta_{1 l} .
$$

Otherwise, if $N \neq 0,-1$, then $S_{I}(l)$ represents the self-interference, which can be approximated as a Gaussian random variable with zero mean and variance expressed as

$$
\operatorname{Var}\left[S_{I}(l)\right]=\frac{\Omega}{3 M}
$$

where $\Omega=E\left[\alpha_{k l}^{2}\right]$.

The multiple-access interference term, due to the $k$ th interfering user given by (12), can be approximated as a Gaussian random variable with zero mean and variance of

$$
\operatorname{Var}\left[M_{I}(k, l)\right]=\frac{\rho \Omega}{3 M} .
$$

Finally, the thermal noise term $\eta_{I}$ given by (14) is a Gaussian random variable with zero mean and variance

$$
\operatorname{Var}\left[\eta_{I}\right]=\frac{1}{2 M \bar{\gamma}_{c}}
$$

where $\bar{\gamma}_{c}=P_{R} T_{c} / N_{0}$ represents the signal-to-noise ratio (SNR) per chip, that is, the SNR/chip.

Consequently, assuming that an $H_{1}$ cell is being tested, according to (9) the normalized variance of $V_{\mathrm{ci}}$ can be expressed as

$$
\operatorname{Var}\left[V_{\mathrm{ci}}\right]=\frac{(L-1) \Omega}{3 M}+\frac{(U-1) L \rho \Omega}{3 M}+\frac{1}{2 M \bar{\gamma}_{c}} .
$$

If we assume that an $H_{0}$ cell is being tested, all $L$ paths from the user-of-interest constitute interference, and consequently, the variance of $V_{\mathrm{ci}}$ can be computed by (19) if we replace the $(L-1)$ term by $L$. However, we assume for simplicity that the variance of $V_{\mathrm{ci}}$ under the $H_{0}$ hypothesis is the same as that given by (19).

The statistics of the quadrature-phase component $V_{\mathrm{si}}$ can also be obtained according to the above method, resulting in

$$
E\left[S_{s}(l)\right]_{N=0}=E\left[S_{S}(l)\right]_{N=-1}=\frac{3}{4} \alpha_{1 l}\left(-\sin \theta_{1 l}\right)
$$

and the normalized variance of $V_{\mathrm{si}}$ is $\operatorname{Var}\left[V_{\mathrm{si}}\right]=\operatorname{Var}\left[V_{\mathrm{ci}}\right]$.

\section{B. Conditional Probability Density Function}

We have derived the statistics of the correlator's outputs, $V_{\mathrm{ci}}$ and $V_{\mathrm{si}}$, for the in-phase branch and the quadrature branch, respectively, which are approximately Gaussian random processes. Hence, for the conventional cell-by-cell detection, the decision variable $Y_{i}=V_{i}=V_{\mathrm{ci}}^{2}+V_{\mathrm{si}}^{2}$ represents either an $H_{1}$ state or an $H_{0}$ state. Given the Gaussian nature of $V_{\mathrm{ci}}$ and $V_{\mathrm{si}}$, and assuming that $V_{i}$ constitutes an $H_{1}$ sample, then $Y_{i}$, for a given $\alpha_{1 l}$, obeys the noncentral chi-square distribution with two degrees of freedom. Its pdf can be expressed according to [18, Eq. $(2-1-121)]$ as

$$
f_{Y_{i}}\left(y \mid H_{1}\right)=\frac{1}{2 \sigma_{o}^{2}} \exp \left(-\frac{m^{2}+y}{2 \sigma_{o}^{2}}\right) I_{0}\left(\frac{m \sqrt{y}}{\sigma_{o}^{2}}\right), \quad y \geq 0
$$

where

$$
\begin{aligned}
\sigma_{o}^{2} & =\operatorname{Var}\left[V_{\mathrm{ci}}\right]=\operatorname{Var}\left[V_{\mathrm{si}}\right] \\
& =\frac{(L-1) \Omega}{3 M}+\frac{(U-1) L \rho \Omega}{3 M}+\frac{1}{2 M \bar{\gamma}_{c}} .
\end{aligned}
$$

$I_{0}()$ is the zeroth-order modified Bessel function of the first kind defined in [18], and $m^{2}$ is the normalized noncentral metric, given by

$$
\begin{aligned}
m^{2} & =\left(E\left[S_{I}(l)\right]_{N=0}\right)^{2}+\left(E\left[S_{s}(l)\right]_{N=0}\right)^{2} \\
& =\left(E\left[S_{I}(l)\right]_{N=-1}\right)^{2}+\left(E\left[S_{s}(l)\right]_{N=-1}\right)^{2} \\
& =\frac{9}{16} \alpha_{1 l}^{2} .
\end{aligned}
$$


When $V_{i}$ constitutes an $H_{0}$ sample, $Y_{i}$ is central chi-square distributed with two degrees of freedom. Its pdf can be expressed according to [18, eq. (2-1-110)] as

$$
f_{Y_{i}}\left(y \mid H_{0}\right)=\frac{1}{2 \sigma_{o}^{2}} \exp \left(-\frac{y}{2 \sigma_{o}^{2}}\right), \quad y \geq 0 .
$$

For the search mode using joint two-cell detection, we have $Y_{i}=V_{i-1}+V_{i}=V_{c(i-1)}^{2}+V_{s(i-1)}^{2}+V_{\mathrm{ci}}^{2}+V_{\mathrm{si}}^{2}$. Hence, $Y_{i}$ has four states, namely, $\left\{H_{0}, H_{1}\right\},\left\{H_{1}, H_{1}\right\},\left\{H_{1}, H_{0}\right\}$, and $\left\{H_{0}, H_{0}\right\}$. Consequently, when $V_{i-1}$ and $V_{i}$ constitute two independent $H_{1}$ samples from the same multipath component, $Y_{i}$ obeys the noncentral chi-square distribution with four degrees of freedom. Its pdf can be expressed according to $[18$, eq. $(2-1-121)]$ as

$$
\begin{gathered}
f_{Y_{i}}^{1}\left(y \mid H_{1}, H_{1}\right) \\
=\frac{1}{2 \sigma_{O}^{2}}\left(\frac{y}{2 m^{2}}\right)^{1 / 2} \exp \left(-\frac{2 m^{2}+y}{2 \sigma_{o}^{2}}\right) I_{1}\left(\frac{m \sqrt{2 y}}{\sigma_{o}^{2}}\right) \\
y \geq 0
\end{gathered}
$$

where $I_{1}(\mathrm{O})$ is the first-order modified Bessel function of the first kind and the noncentral parameter $2 \mathrm{~m}^{2}$ is contributed by two $H_{1}$ samples from the same path, where $m^{2}$ is given by (23). If $V_{i-1}$ and $V_{i}$ constitute two independent $H_{1}$ samples from two different propagation paths, the pdf of $Y_{i}$ can be expressed according to [18, Eq. (2-1-121)] as

$$
\begin{gathered}
f_{Y_{i}}^{2}\left(y \mid H_{1}, H_{1}\right) \\
=\frac{1}{2 \sigma_{o}^{2}}\left(\frac{y}{m^{\prime 2}}\right)^{1 / 2} \exp \left(-\frac{m^{\prime 2}+y}{2 \sigma_{o}^{2}}\right) I_{1}\left(\frac{m^{\prime} \sqrt{y}}{\sigma_{o}^{2}}\right) \\
y \geq 0
\end{gathered}
$$

where the noncentral parameter $m^{\prime 2}$ is contributed by two independent paths and given by

$$
m^{\prime 2}=\frac{9}{16}\left(\alpha_{1 l_{1}}^{2}+\alpha_{1 l_{2}}^{2}\right) .
$$

When $V_{i-1}$ constitutes an $H_{0}$ sample and $V_{i}$ an $H_{1}$ sample, or conversely, $V_{i-1}$ an $H_{1}$ sample and $V_{i}$ an $H_{0}$ sample, the pdf of $Y_{i}$ can be computed by straightforward convolution of the pdfs of $V_{i-1}$ and $V_{i}$, which results in

$$
\begin{aligned}
& f_{Y_{i}}\left(y \mid H_{0}, H_{1}\right) \\
&= f_{Y_{i}}\left(y \mid H_{1}, H_{0}\right) \\
&= \frac{1}{2 \sigma_{o}^{2}}\left(\frac{y}{m^{2}}\right)^{1 / 2} \exp \left(-\frac{m^{2}+y}{2 \sigma_{o}^{2}}\right) I_{1}\left(\frac{m \sqrt{y}}{\sigma_{o}^{2}}\right) \\
& y \geq 0 .
\end{aligned}
$$

Finally, assuming that $V_{i-1}$ and $V_{i}$ constitute two $H_{0}$ samples, the pdf of $Y_{i}$ is the central chi-square distribution with four degrees of freedom, which can be expressed according to [18, Eq. (2-1-110)] as

$$
f_{Y_{i}}\left(y \mid H_{0}, H_{0}\right)=\frac{y}{4 \sigma_{o}^{4}} \exp \left(-\frac{y}{2 \sigma_{o}^{2}}\right), \quad y \geq 0 .
$$

Above, we have derived the statistics of the decision variables under different hypotheses. Let us now derive the overall miss probability and the false-alarm probability.

\section{Miss PROBABILITY AND FALSE-AlaRm PROBABILITY}

In this section, we first derive the overall miss probability for the serial search in conjunction with conventional cell-by-cell detection or joint two-cell detection under the condition of given instantaneous fading attenuation of the dispersive paths, namely, for $\alpha_{1 l}, l=1,2, \ldots, L$. Then, the average probabilities are derived by averaging the corresponding conditional probabilities over the dynamic range of $\alpha_{1 l}, l=1,2, \ldots, L$. Let us first consider the conventional cell-by-cell detection.

\section{A. Conventional Cell-by-Cell Detection}

For the conventional cell-by-cell detection, each $H_{1}$ cell associated with different propagation paths has the same statistics, and similarly, each $H_{0}$ cell related to different paths has the same statistics. Their statistics can be described by (21) and (24), respectively. Hence, the detection probability of an $H_{1}$ cell for a certain value of $\alpha_{1 l}$ is formulated as

$$
P_{D}(S)=\int_{h}^{\infty} f_{Y_{i}}\left(y \mid H_{1}\right) d y=Q\left(S, \sqrt{h^{\prime}}\right)
$$

where $f_{Y_{i}}\left(y \mid H_{1}\right)$ is given by (21). Furthermore, $h^{\prime}=h / \sigma_{o}^{2}$ is the normalized threshold, $Q(a, b)=Q_{1}(a, b)$ represents Marcum's $Q$ function as defined in [18, eq. (2-1-122)], and

$$
S=\sqrt{\frac{m^{2}}{\sigma_{o}^{2}}}=S_{0} \cdot \frac{\alpha_{1 l}}{\sqrt{\Omega}}
$$

with $S^{2}$ being the conditional SNR per path over the $M$-chip integration dwell time conditioned on the instantaneous fading parameter $\alpha_{1 l}$, where $S_{0}$ is expressed as

$$
S_{0}=\frac{3}{4}\left(\frac{L-1}{3 M}+\frac{(U-1) L \rho}{3 M}+\frac{1}{2 M \Omega \bar{\gamma}_{c}}\right)^{-1 / 2}
$$

and $S_{0}^{2}$ is the average SNR per path over $M$ chips.

The detection probability $P_{D}(S)$ given in (30) is conditioned on the SNR of $S^{2}$ per path integrated over $M$ chips, and consequently through (31) conditioned on the distribution of $\alpha_{1 l}$, representing the $l$ th fading diversity path amplitude. The (unconditional) average detection probability of an $H_{1}$ sample can be computed by averaging (30) over the range of $S$. Since $\alpha_{1 l}$ is Rayleigh-distributed with its pdf given by (7), the pdf of $S$ in (31) can be readily expressed with the aid of (7) as

$$
f_{S}(s)=\frac{2 s}{S_{0}^{2}} \exp \left(-\frac{s^{2}}{S_{0}^{2}}\right), \quad s \geq 0 .
$$

Finally, using the result of the Appendix, we can obtain the average detection probability of an $H_{1}$ sample by setting $\mu=$ $1, p=1, \sigma^{2}=S_{o}^{2} / 2$, and $b^{2}=h^{\prime}$ in (65), resulting in

$$
P_{D}=\sum_{i=0}^{\infty} \sum_{j=0}^{i} \frac{\left(h^{\prime}\right)^{i-j}}{(i-j) ! 2^{i-j-1}} \cdot \frac{\exp \left(-h^{\prime} / 2\right)}{S_{0}^{2}\left(1+2 / S_{0}^{2}\right)^{i+1}} .
$$


We have assumed that the statistics of different propagation paths of the desired signal are independent and that there are $L$ paths, where each path is associated with two $H_{1}$ cells. Hence, the $L$-path sequences of the user-of-interest presented to the receiver can be modeled as a sequence with a total uncertainty region of $q+2(L-1)$, where $q$ is the uncertainty region of the transmitted sequence, while the $2(L-1)$ term is a consequence of the $L$-path channel spreading due to the multipath fading. Furthermore, since each of the $L$ paths is associated with two $H_{1}$ cells, there is a total of $2 L H_{1}$ cells. Therefore, the overall miss probability defined in Section III can be expressed as

$$
P_{M}(2 L)=\left(1-P_{D}\right)^{2 L}
$$

where $P_{D}$ is given by (34).

The false-alarm probability is defined the probability that the output decision variable $Y_{i}$ under the hypothesis $H_{0}$ exceeds the threshold $h$. This can be expressed as

$$
P_{\mathrm{FA}}=\int_{h}^{\infty} f_{Y_{i}}\left(y \mid H_{0}\right) d y=\exp \left(-\frac{h^{\prime}}{2}\right) .
$$

With the aid of our results generated so far, we can now evaluate the MAT performance $\bar{T}_{\text {acq }}$ of the serial search mode using conventional cell-by-cell detection by substituting the overall miss probability of (35) as well as the false-alarm probability of (36) and the other related parameters into (4).

\section{B. Joint Two-Cell Detection}

Since the $i$ th sample is either $H_{1}$ or $H_{0}$, for the joint two-cell detection the decision variable $Y_{i}$, which is derived from $\left\{V_{i-1}, V_{i}\right\}$, has four possible phase states, i.e., $D_{1}=\left\{H_{0}, H_{1}\right\}, D_{2}=\left\{H_{1}, H_{0}\right\}, D_{3}=\left\{H_{1}, H_{1}\right\}$, and $F=\left\{H_{0}, H_{0}\right\}$. Furthermore, the two adjacent $T_{c} / 2$-spaced samples $V_{i-1}$ and $V_{i}$ in Fig. 1 can be the samples from the same propagation path or from two different paths.

Let states $D_{1}, D_{2}$, and $D_{3}$ represent the correct states, while $F$ represent the incorrect state. We assume that the acquisition process terminates and successfully proceeds to the tracking process if the two adjacent samples belong to $D_{1}, D_{2}$, or $D_{3}$ and the corresponding output decision variable $Y_{i}=V_{i-1}+V_{i}$ exceeds the threshold $h$ in Fig. 1. Similarly, a false alarm occurs if the two adjacent samples belong to $F$ and the corresponding output decision variable $Y_{i}=V_{i-1}+V_{i}$ exceeds the detection threshold $h$.

For the joint two-cell detection scenario, we commence by considering the false-alarm probability. Assuming that the current detection is only influenced by the previous two cells, and that the correlation with the other cells can be neglected, then it can be shown that the false-alarm probability at the $n$th incorrect state can be written as

$$
\begin{aligned}
& \alpha=P\left(Y_{n}>h\right) \\
& =P\left(V_{n}>h\right)+P\left(Y_{n-2}>h\right) P\left(V_{n-1}<h, V_{n}<h,\right. \\
& \left.\quad Y_{n}>h\right)+P\left(V_{n-2}<h, Y_{n-1}<h, V_{n}<h, Y_{n}>h\right) .
\end{aligned}
$$

Let us assume that the serial search acquisition process is a stationary stochastic process. Then, we have $P\left(Y_{n-2}>h\right)=$
$P\left(Y_{n}>h\right)=\alpha$. Replacing $P\left(Y_{n-2}>h\right)$ by $\alpha$ in the above equation, we obtain

$$
\frac{\alpha=}{P\left(V_{n}>h\right)+P\left(V_{n-2}<h, Y_{n-1}<h, V_{n}<h, Y_{n}>h\right)}
$$

where $P\left(V_{n}>h\right)$ is given by (36). The second term in the numerator of (38) can be expressed as

$$
\begin{aligned}
& P\left(V_{n-2}<h, Y_{n-1}<h, V_{n}<h, Y_{n}>h\right) \\
& =\int_{0}^{h} f_{V_{n-1}}\left(y \mid H_{0}\right)\left[\int_{0}^{h-y} f_{V_{n-2}}\left(x \mid H_{0}\right) d x\right] \\
& \quad \times\left[\int_{h-y}^{h} f_{V_{n}}\left(x \mid H_{0}\right) d x\right] d y .
\end{aligned}
$$

Upon using the corresponding pdfs in the above equation, we can simplify it to

$$
\begin{aligned}
& P\left(V_{n-2}<h, Y_{n-1}<h, V_{n}<h, Y_{n}>h\right) \\
& \quad=\left(\frac{h^{\prime}}{2}-2\right) \exp \left(-\frac{h^{\prime}}{2}\right)+\left(\frac{h^{\prime}}{2}+2\right) \exp \left(-h^{\prime}\right) .
\end{aligned}
$$

The second term in the denominator of (38) can be expressed as

$$
\begin{aligned}
P & \left(V_{n-1}<h, V_{n}<h, Y_{n}>h\right) \\
& =\int_{0}^{h} f_{V_{n-1}}\left(y \mid H_{0}\right)\left[\int_{h-y}^{h} f_{V_{n}}\left(x \mid H_{0}\right) d x\right] d y \\
& =\left(\frac{h^{\prime}}{2}-1\right) \exp \left(-\frac{h^{\prime}}{2}\right)+\exp \left(-h^{\prime}\right) .
\end{aligned}
$$

Upon substituting (36), (40), and (41) into (38), finally, the false alarm probability can be expressed as

$$
\alpha=\frac{\left(\frac{h^{\prime}}{2}-1\right) \exp \left(-\frac{h^{\prime}}{2}\right)+\left(\frac{h^{\prime}}{2}+2\right) \exp \left(-h^{\prime}\right)}{1-\left(\frac{h^{\prime}}{2}-1\right) \exp \left(-\frac{h^{\prime}}{2}\right)-\exp \left(-h^{\prime}\right)} .
$$

Let us assume that there are $L$ resolvable paths at the base station. As we discussed in the context of the conventional cell-by-cell detection scenario, the frequency-selective faded sequence of the user-of-interest presents itself to the BS as a sequence with a total of $q+2(L-1)$ cells in the uncertainty region. For joint two-cell detection, the frequency-selective faded sequence can be approximated as a sequence with a total of $q+2(L-1)$ number of states and $2 L+1$ correct states. The $2 L+1$ number of correct states include one $\left\{H_{0}, H_{1}\right\}$, one $\left\{H_{1}, H_{0}\right\}, L$ number of $\left\{H_{1}, H_{1}\right\}$ states associated with two $H_{1}$ samples from the same path, and $L-1$ number of $\left\{H_{1}, H_{1}\right\}$ states associated with two $H_{1}$ samples from two different paths. Consequently, the overall miss probability of the serial search mode can be expressed as

$$
\begin{aligned}
& P_{M}(2 L+1) \\
& =E\left\{P \left(Y_{1}=\left(V_{0}+V_{1}^{1}\right)<h, Y_{2}=\left(V_{1}^{1}+V_{2}^{1}\right)<h\right.\right. \\
& Y_{3}=\left(V_{2}^{1}+V_{3}^{2}\right)<h, Y_{4}=\left(V_{3}^{2}+V_{4}^{2}\right) \\
& \quad<h, \ldots, Y_{2 L-1}=\left(V_{2 L-2}^{L-1}+V_{2 L-1}^{L}\right)<h \\
& Y_{2 L}=\left(V_{2 L-1}^{L}+V_{2 L}^{L}\right)<h \\
& \left.\left.Y_{2 L+1}=\left(V_{2 L}^{L}+V_{0}\right)<h\right)\right\}
\end{aligned}
$$


where $V_{0}$ represents an $H_{0}$ sample, while the other $\left\{V_{i}^{j}\right\}$ variables in the above equation represent $H_{1}$ samples. $Y_{2 i-1}=$ $V_{2 i-2}^{i-1}+V_{2 i-1}^{i}$ represents the sum of two $H_{1}$ samples from the $(i-1)$ th and $i$ th paths, $Y_{2 i}=V_{2 i-1}^{i}+V_{2 i}^{i}$ represents two $H_{1}$ samples from the $i$ th path, and $E[\cdot]$ represents the average over the fading parameters.

However, for the search mode using joint two-cell detection, due to the correlation between two adjacent decision variables, i.e., between $Y_{2 i-1}$ and $Y_{2 i}$, it is arduous to derive the closed-form expression of the overall miss probability. Hence, here we aim to derive its lower and upper bound. The lower bound of the overall miss probability is derived by ignoring the dependence between the two adjacent decision variables, yielding

$$
\begin{aligned}
& P_{M}(2 L+1) \\
& \geq E\left\{P\left(Y_{1}=\left(V_{0}+V_{1}^{1}\right)<h\right)\right\} \\
& \quad \times \prod_{i=1}^{L} E\left\{P\left(Y_{2 i}=\left(V_{2 i-1}^{i}+V_{2 i}^{i}\right)<h\right)\right\} \\
& \quad \cdot \prod_{j=1}^{L-1} E\left\{P\left(Y_{2 j+1}=\left(V_{2 j}^{j}+V_{2 j+1}^{j+1}\right)<h\right)\right\} \\
& \quad \times E\left\{P\left(Y_{2 L+1}=\left(V_{2 L}^{L}+V_{0}\right)<h\right)\right\}
\end{aligned}
$$

where the terms taking the form of $E\left[P\left(Y_{i}<h\right)\right]$ represent the related average miss probabilities of the correct states. The relevant terms in (44) can be obtained by determining the related detection probabilities first as follows. If $V_{0}$ is an $H_{0}$ sample and $V_{1}^{1}$ is an $H_{1}$ sample, which corresponds to the first term of (44), or conversely, $V_{2 L}^{L}$ is an $H_{1}$ sample and $V_{0}$ is an $H_{0}$ sample, which corresponds to the last term of (44), then the detection probability conditioned on the fading amplitude $\alpha_{1 l}$ of path $l$ can be expressed as

$$
\begin{aligned}
P_{D_{1}}(S) & =P_{D_{2 L+1}}(S) \\
& =\int_{h}^{\infty} f_{Y_{i}}\left(y \mid H_{1}, H_{0}\right) d y=Q_{2}\left(S, \sqrt{h^{\prime}}\right)
\end{aligned}
$$

where $f_{Y_{i}}\left(y \mid H_{1}, H_{0}\right)$ is given by (28), $Q_{2}(a, b)$ represents Marcum's $Q$ function defined by [18, Eq. (2-1-122) ], $h^{\prime}=h / \sigma_{o}^{2}$ is the normalized threshold as we defined previously, and $S$ is defined in (31).

The unconditional detection probability of state $D_{1}$ or $D_{2 L+1}$ can be obtained by averaging (45) over the range of $S$, which can be inferred from (65) of the Appendix by setting $\mu=2, p=$ $1, \sigma^{2}=S_{0}^{2} / 2$, and $b^{2}=h^{\prime}$, yielding

$$
\begin{aligned}
P_{D_{1}} & =P_{D_{2 L+1}} \\
& =\sum_{m=0}^{\infty} \sum_{n=0}^{m+1} \frac{\left(h^{\prime}\right)^{m-n+1}}{(m-n+1) ! 2^{m-n}} \cdot \frac{\exp \left(-h^{\prime} / 2\right)}{S_{0}^{2}\left(1+2 / S_{0}^{2}\right)^{m+1}} .
\end{aligned}
$$

Similarly, if $V_{2 i-1}^{i}$ and $V_{2 i}^{i}$ are two $H_{1}$ samples from the same path, a situation that corresponds to the second term of (44), then the conditional detection probability for $i=1,2, \ldots, L$ can be expressed as

$$
P_{D_{2 i}}(S)=\int_{h}^{\infty} f_{Y_{2 i}^{1}}\left(y \mid H_{1}, H_{1}\right) d y=Q_{2}\left(\sqrt{2} S, \sqrt{h^{\prime}}\right)
$$

where $f_{Y_{2 i}^{1}}\left(y \mid H_{1}, H_{1}\right)$ is given by (25), while $S$ is given by (31). The unconditional detection probability can be similarly computed from (65) of the Appendix by setting $\mu=2, p=$ $1, \sigma^{2}=S_{0}^{2}$ and $b^{2}=h^{\prime}$, which results in

$$
\begin{aligned}
P_{D_{2 i}}= & \sum_{m=0}^{\infty} \sum_{n=0}^{m+1} \frac{\left(h^{\prime}\right)^{m-n+1}}{(m-n+1) ! 2^{m-n+1}} \\
& \cdot \frac{\exp \left(-h^{\prime} / 2\right)}{S_{0}^{2}\left(1+1 / S_{0}^{2}\right)^{m+1}}, \quad i=1,2, \ldots, L .
\end{aligned}
$$

If $V_{2 j}^{j}$ and $V_{2 j+1}^{j+1}$ are two $H_{1}$ samples from two different paths, a scenario that corresponds to the third term of (44), then the detection probability conditioned on $\alpha_{1 l_{1}}$ and $\alpha_{1 l_{2}}$ can be expressed as

$$
P_{D_{2 j+1}}\left(S^{\prime}\right)=\int_{h}^{\infty} f_{Y_{2 j+1}^{2}}\left(y \mid H_{1}, H_{1}\right) d y=Q_{2}\left(S^{\prime}, \sqrt{h^{\prime}}\right)
$$

where $f_{Y_{2 j+1}^{2}}\left(y \mid H_{1}, H_{1}\right)$ is given by (25), while $S^{\prime}$ is given by

$$
S^{\prime}=S_{0} \cdot \sqrt{\frac{\alpha_{1 l_{1}}^{2}+\alpha_{1 l_{2}}^{2}}{\Omega}} .
$$

Since $\alpha_{1 l_{1}}$ and $\alpha_{1 l_{2}}$ are two i.i.d. Rayleigh random variables, the pdf of $S^{\prime}$ can be derived using (63) of the Appendix with $p=2$, which can be expressed as

$$
f_{S}^{\prime}(s)=\frac{2 s^{3}}{S_{0}^{4}} \exp \left(-\frac{s^{2}}{S_{0}^{2}}\right), \quad s \geq 0 .
$$

The unconditional detection probability can be obtained by averaging (49) using (51) in the valid range of $S^{\prime}$, which can also be obtained from (65) of the Appendix by setting $\mu=2, p=$ $2, \sigma^{2}=S_{0}^{2} / 2$, and $b^{2}=h^{\prime}$, yielding

$$
\begin{aligned}
P_{D_{2 j+1}}= & \sum_{m=0}^{\infty} \sum_{n=0}^{m+1} \frac{(m+1)\left(h^{\prime}\right)^{m-n+1}}{(m-n+1) ! 2^{m-n-1}} \\
& \cdot \frac{\exp \left(-h^{\prime} / 2\right)}{S_{0}^{4}\left(1+2 / S_{0}^{2}\right)^{m+2}}, \quad j=1,2, \ldots, L-1 .
\end{aligned}
$$

Consequently, with the aid of (46), (48), and (52), the overall miss probability of the serial search mode using joint two-cell detection obeys

$$
\begin{aligned}
P_{M}(2 L+1) \geq & \left(1-P_{D_{1}}\right)\left(1-P_{D_{2 L+1}}\right)\left(1-P_{D_{2 i}}\right)^{L} \\
& \times\left(1-P_{D_{2 j+1}}\right)^{L-1} .
\end{aligned}
$$

The upper bound of the overall miss probability for the joint two-cell detection can be achieved if we set $V_{0}$ and $V_{2 i-2}^{i-1}$ in $Y_{2 i-1}$ of (43) equal to zero for $i=2,3, \ldots, L$, i.e.,

$$
\begin{aligned}
P_{M}(2 L+1) \leq & E\left\{P \left(V_{1}^{1}<h, Y_{2}=V_{1}^{1}+V_{2}^{1}<h ;\right.\right. \\
& V_{3}^{2}<h, Y_{4}=V_{3}^{2}+V_{4}^{2}<h ; \ldots \\
& \left.\left.V_{2 L-1}^{L}<h, Y_{2 L}=V_{2 L-1}^{L}+V_{2 L}^{L}<h\right)\right\} .
\end{aligned}
$$

Note that (54) corresponds to the overall miss probability of the search mode, where we assume that the delayed sample is set to zero, when the "present" sample is due to different path of the delayed sample. Since on the right-hand side of (54) the 
$\left(V_{2 i-1}^{i}<h, Y_{2 i}=V_{2 i-1}^{i}+V_{2 i}^{i}<h\right)$ terms for $i=1,2, \ldots, L$ are mutually independent, (54) can be written as

$$
\begin{aligned}
& P_{M}(2 L+1) \\
& \quad \leq \prod_{l=1}^{L} E\left\{P\left(V_{2 i-1}^{i}<h, Y_{2 i}=\left(V_{2 i-1}^{i}+V_{2 i}^{i}\right)<h\right)\right\} \\
& \quad=\prod_{l=1}^{L} E\left\{P\left(Y_{2 i}<h\right)\right\}
\end{aligned}
$$

where $P\left(Y_{2 i}<h\right)$ is the probability that the decision variable $Y_{2 i}$ does not exceed the threshold of $h$. Since $Y_{2 i}=V_{2 i-1}^{i}+V_{2 i}^{i}$, where $V_{2 i-1}^{i}$ and $V_{2 i}^{i}$ represent two $H_{1}$ samples from the same path, $P\left(Y_{2 i}<h\right)$ can be expressed as

$$
\begin{aligned}
P\left(Y_{2 i}<h\right) & =\int_{0}^{h} f_{Y_{2 i}}^{1}\left(y \mid H_{1}, H_{1}\right) d y \\
& =1-Q_{2}\left(\sqrt{2} S, \sqrt{h^{\prime}}\right)
\end{aligned}
$$

where $f_{Y_{2 i}}^{1}\left(y \mid H_{1}, H_{1}\right)$ is given by (25), while $S$ is given by (31). The corresponding unconditional detection miss probability can be computed with the aid of (65) in the Appendix by setting $\mu=2, p=1, \sigma^{2}=S_{0}^{2}$, and $b^{2}=h^{\prime}$, which results in

$$
\begin{aligned}
E\left[P\left(Y_{2 i}<h\right)\right]= & 1-\sum_{m=0}^{\infty} \sum_{n=0}^{m+1} \frac{\left(h^{\prime}\right)^{m-n+1}}{(m-n+1) ! 2^{m-n+1}} \\
& \cdot \frac{\exp \left(-h^{\prime} / 2\right)}{S_{0}^{2}\left(1+1 / S_{0}^{2}\right)^{m+1}}
\end{aligned}
$$

for $i=1,2, \ldots, L$.

Substituting (57) into (55), finally, the overall miss probability of the serial search scheme using joint two-cell detection is given by

$$
\begin{aligned}
P_{M}(2 L+1) \leq\left[1-\sum_{m=0}^{\infty} \sum_{n=0}^{m+1} \frac{\left(h^{\prime}\right)^{m-n+1}}{(m-n+1) ! 2^{m-n+1}}\right. \\
\left.\cdot \frac{\exp \left(-h^{\prime} / 2\right)}{S_{0}^{2}\left(1+1 / S_{0}^{2}\right)^{m+1}}\right]^{L} .
\end{aligned}
$$

Finally, the lower bound and upper bound MAT of the serial search mode using joint two-cell detection can be evaluated by substituting (42) and (53) as well as (58) and the other related parameters in (4). Let us now evaluate the performance of the schemes studied.

\section{NUMERICAL RESULTS AND COMPARISONS}

In this section, the MAT performance of the serial search scheme using conventional cell-by-cell detection and joint two-cell detection was evaluated and compared. As an application for the serial search using the above two detection schemes, we consider a periodic PN sequence of length 1023. Consequently, $q$, which determines the length of the uncertainty region for the serial search mode, is 2046, since the search step size is $T_{c} / 2$. For convenience, we consider the normalized MAT, which is derived from the MAT given by (4) divided by the bit duration of $T=G T_{c}$, when different overall miss probabilities and different false alarm probabilities are considered. The associated parameters are shown at the top of the figures. Note that three different SNRs are used in our analysis, namely,

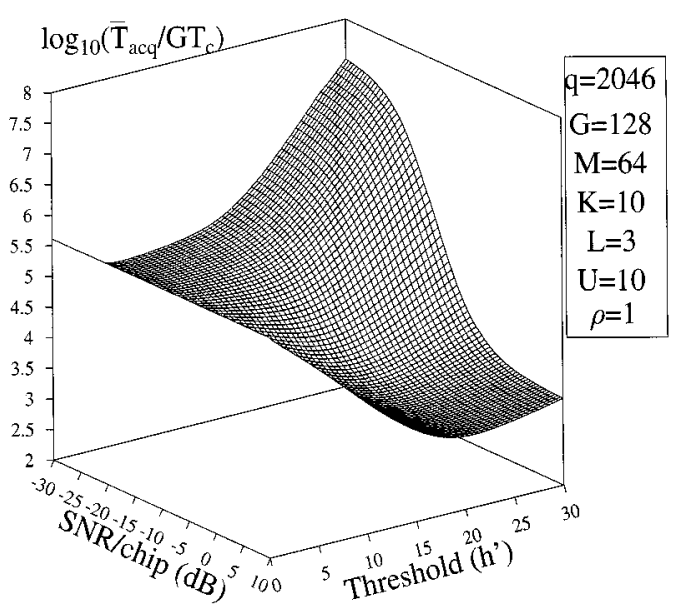

Fig. 4. MAT versus SNR/chip and normalized threshold $h^{\prime}$ performance for the serial search scheme using conventional cell-by-cell detection computed from (4), (35), and (36).

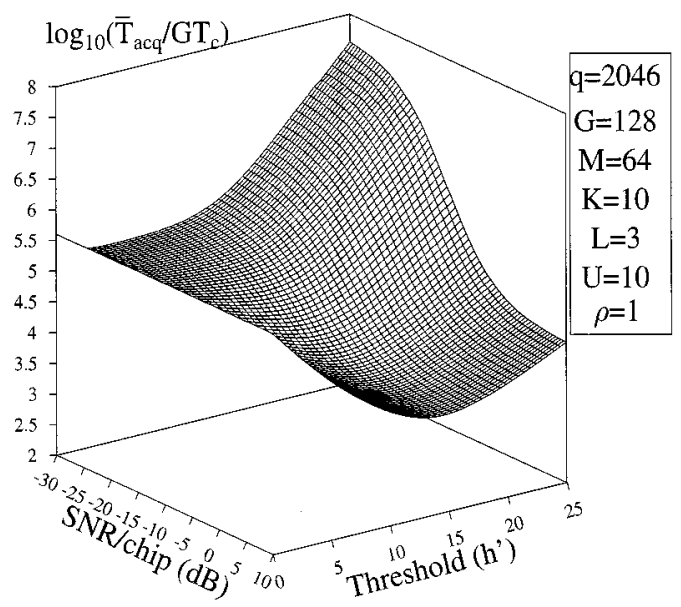

Fig. 5. MAT versus SNR/chip and normalized threshold $h^{\prime}$ performance for the serial search scheme using joint two-cell detection computed from (4), (42), and (53).

SNR/chip, SNR per $M$ chips, i.e., the energy over the integral dwell time $\left(\tau_{D}=M T_{c}\right.$ s) to noise ratio, and the SNR/bit.

Figs. 4 and 5 show the normalized MAT performance versus the normalized threshold $h^{\prime}$ and the SNR/chip for the serial search modes using conventional cell-by-cell detection (Fig. 4) and joint two-cell detection (Fig. 5) (lower bound), respectively. It is clear from Figs. 4 and 5 that an inappropriate choice of the detection threshold $h^{\prime}$ can lead to severe increase of the MAT, but the sensitivity of the MAT to the threshold decreases as the SNR/chip increases. For any given SNR/chip value, there exists an optimal choice of the threshold $h^{\prime}$, which minimizes the MAT. In addition, for any given normalized threshold $h^{\prime}$, the MAT decreases as the SNR/chip increases, and finally reaches a residual value, which is essentially due to the false-alarm probability and the associated "penalty" time incurred before reverting back to the search mode. This value can be computed from (4) by setting $P_{M}(\lambda)=0$, resulting in $\bar{T}_{\text {acq }} \approx(1+K \alpha) / 2 \cdot\left(q \tau_{D}\right)$.

In Fig. 6, we evaluate and compare the MAT versus SNR/chip performance of both conventional cell-by-cell detection and 


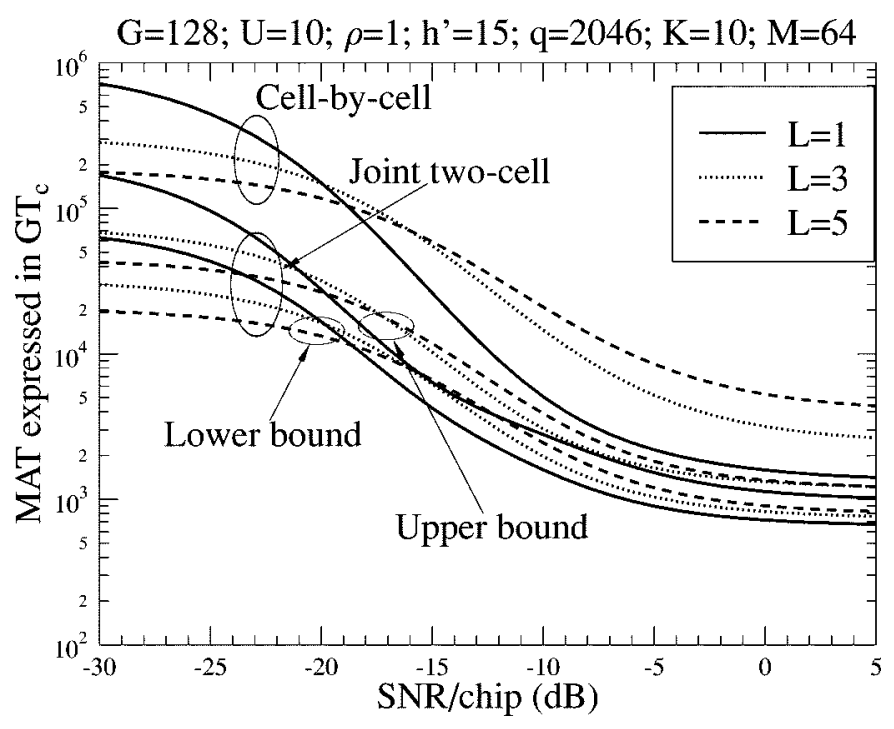

Fig. 6. MAT expressed in bit durations, i.e., $T=G T_{c}$ versus SNR/chip performance for the serial search scheme using conventional cell-by-cell detection and joint two-cell detection computed from (4), (35), (36), (42), (53), and (58).

joint two-cell detection parameterized with the number of resolvable paths. From the results we notice that for low SNR/chip values, an increased number of resolvable paths can decrease the MAT. However, for high SNR/chip values, a high number of resolvable paths increases the MAT above the crossover zone near $-17 \mathrm{~dB}$. If we assume that a practical acquisition system operates at an average SNR/bit in the range of 5-20 dB, which implies that the average SNR/chip is in the range of -16 to $-1 \mathrm{~dB}$, then it can be concluded that for this type of acquisition system a high number of resolvable paths associated with severe dispersion will degrade the MAT performance. From the results, we find that the MAT performance of the joint two-cell detection is about an order of magnitude better than that of the conventional cell-by-cell detection. Moreover, the MAT performance of joint two-cell detection is more robust to the variation in the number of resolvable paths for a given threshold and for a given SNR/chip than that of the conventional cell-by-cell detection.

Fig. 7 presents the MAT performance of the above two detection methods against the normalized threshold $h^{\prime}$. For any given number of resolvable paths at the BS, and for a given SNR/chip, there is an optimal choice of the threshold $h^{\prime}$, which leads to the minimum MAT. At the optimal value of $h^{\prime}$, we notice that for the conventional cell-by-cell detection or joint two-cell detection the MAT performance is improved, although not dramatically, when the number of resolvable paths increases. However, if the value of the threshold is set inappropriately, especially when it is set above its optimum value, the MAT will significantly increase, as the number of resolvable paths increases. However, as we noticed in Fig. 6, the MAT performance of joint two-cell detection improves more significantly and becomes also more robust to the number of resolvable paths in a wide range of the normalized threshold $h^{\prime}$ from about 7 to 25 than that of conventional cell-by-cell detection assuming a given number of resolvable paths at the BS.

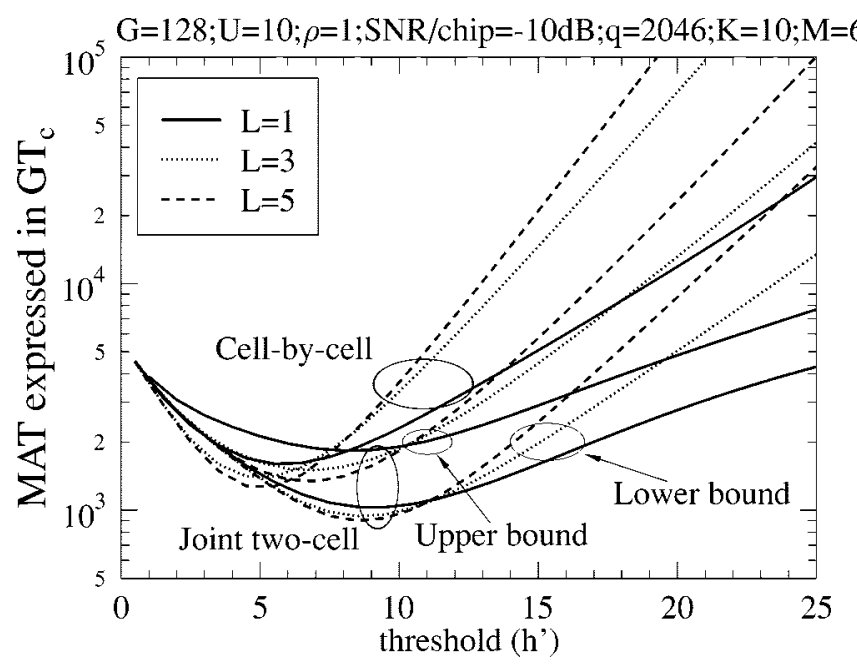

Fig. 7. MAT expressed in bit durations, i.e., $T=G T_{c}$ versus the normalized threshold $h^{\prime}$ performance for the serial search scheme using conventional cell-by-cell detection and joint two-cell detection computed from (4), (35), (36), (42), (53), and (58).

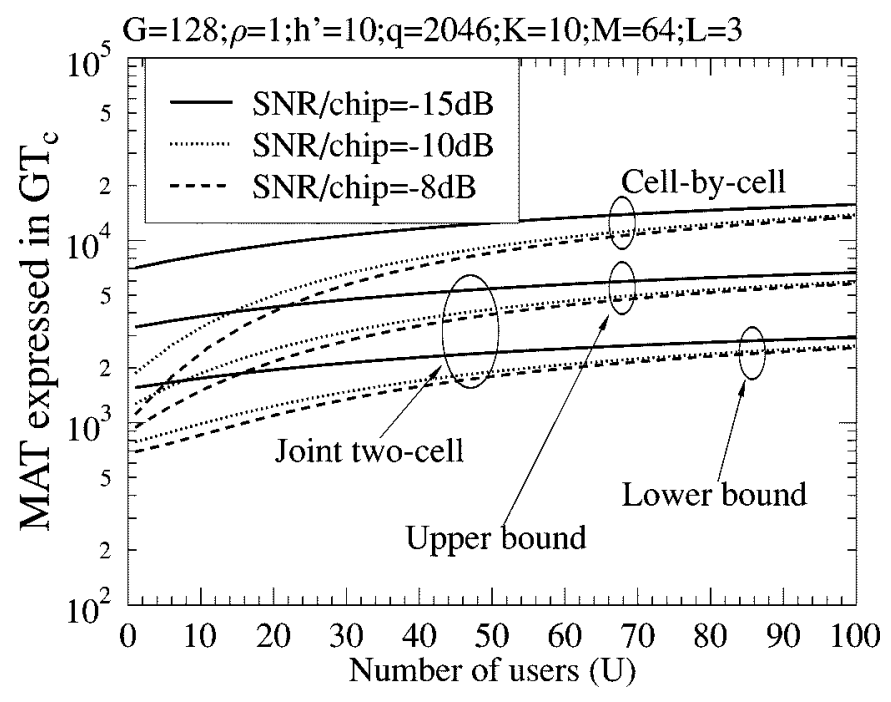

Fig. 8. MAT expressed in bit durations, i.e., $T=G T_{c}$ versus the number of users $U$ performance for the serial search scheme using conventional cell-by-cell detection and joint two-cell detection computed from (4), (35), (36), (42), (53), and (58).

The effect of the number of active users on the MAT performance of both conventional cell-by-cell detection and joint two-cell detection is shown in Fig. 8. As expected, the MAT using both detection schemes increases when the number of active users increases.

Finally, in Figs. 9 and 10, we evaluate the effect of varying the received power of the user-of-interest on the MAT performance for both the conventional cell-by-cell detection and the joint two-cell detection schemes. The curves of Fig. 9 were computed against the SNR/chip value, while the curves of Fig. 10 were computed against the number of active users $U$. From the results, we observe that in the SNR/chip range from -16 to $-1 \mathrm{~dB}$, the MAT performance of both detection schemes was explicitly improved when we increased the power of the user-of-interest. Moreover, it can be seen that the MAT performance of the joint two-cell detection is more robust to the 
$\mathrm{G}=128 ; \mathrm{U}=10 ; \mathrm{h}^{\prime}=10 ; \mathrm{q}=2046 ; \mathrm{K}=10 ; \mathrm{M}=64 ; \mathrm{L}=5$

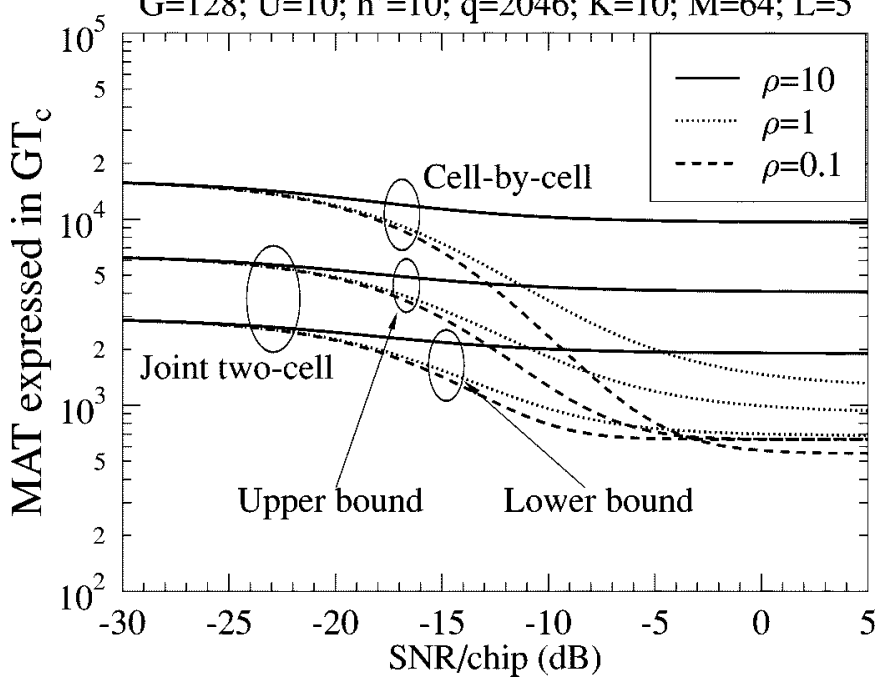

Fig. 9. MAT expressed in bit durations, i.e., $T=G T_{c}$ versus SNR/chip performance for the serial search scheme using conventional cell-by-cell detection and joint two-cell detection computed from (4), (35), (36), (42), (53), and (58).

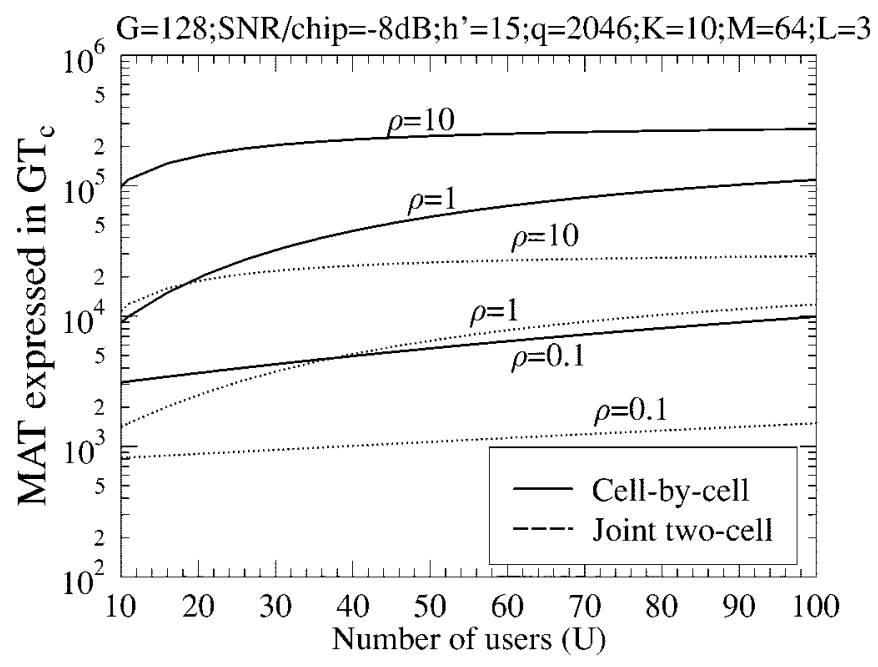

Fig. 10. MAT expressed in bit durations, i.e., $T=G T_{c}$ versus the number of users $U$ performance for the serial search scheme using conventional cell-by-cell detection and joint two-cell detection computed from (4), (35), (36), (42), and (53) parameterized by $\rho$, the ratio of the interfering user's power to that of the user-of-interest.

change of the power of the user-of-interest than that of the conventional cell-by-cell detection. However, from a system capacity point of view, increasing the power of the initial synchronization users implies increasing the interference inflicted upon the data transmission users, which consequently decreases the capacity of the system. Hence, in DS-CDMA systems, an increase in the initial synchronization user's transmitted power in order to aid acquisition should consider the tradeoffs between the MAT and the system capacity.

\section{CONCLUding REMARKS}

A DS-CDMA serial search acquisition system using conventional cell-by-cell detection or joint two-cell detection has been proposed and characterized. A generalized asymptotic expression for computing the MAT in terms of the overall miss probability and the false-alarm probability has been derived. The MAT performance of the serial search system using conventional cell-by-cell detection and that of using joint two-cell detection has been estimated and compared when a frequency-selective multipath fading channel is considered. The analysis presented has utilized practical assumptions and is applicable to the mobile-to-base-station link of a mobile DS-CDMA communication system using BPSK modulation. The effects of multiple-access interference, the number of resolvable paths, and the received power of the initial synchronization user have been investigated. From the results, we conclude that the MAT performance of the serial search acquisition system using joint two-cell detection is more robust to multiple-access interference, to the detection threshold, to the number of resolvable paths, and to the received power of the user-of-interest than that of conventional cell-by-cell detection. Moreover, the MAT performance of joint two-cell detection is found to be better than that of conventional cell-by-cell detection. This is because joint two-cell detection is capable of efficiently combine the energy of two adjacent correct phase samples for making decisions. Our approach can be extended to using the sum of the adjacent $2 L$ samples for detection if we assume that $L$ resolvable paths can be obtained at the receiver, which is the subject of our future research.

\section{APPENDIX}

In this Appendix, we evaluate the integral

$$
\Theta(\sigma, b)=\int_{0}^{\infty} Q_{\mu}(r, b) p_{R}(r) d r
$$

which is often invoked in the main body of this paper. $Q_{\mu}(r, b)$ is the generalized Marcum $Q$-function, which is defined as [18]

$$
Q_{\mu}(r, b)=\int_{b}^{\infty} x\left(\frac{x}{r}\right)^{\mu-1} \exp \left(-\frac{x^{2}+r^{2}}{2}\right) I_{\mu-1}(r x) d x
$$

where $I_{\alpha}(x)$ is the $\alpha$ th order modified Bassel function of the first kind defined as [18]

$$
I_{\alpha}(x)=\sum_{i=0}^{\infty} \frac{(x / 2)^{\alpha+2 i}}{i ! \Gamma(\alpha+i+1)}, \quad x \geq 0 .
$$

In (59) $R$ is a random variable, which is defined as

$$
R=\sqrt{\sum_{m=1}^{2 p} X_{m}^{2}}
$$

where the $X_{m}, m=1,2, \ldots, 2 p$ are statistically independent, identically distributed zero-mean Gaussian random variables. The pdf of $R$ is given by [18]

$$
p_{R}(r)=\frac{r^{2 p-1}}{2^{p-1} \sigma^{2 p}(p-1) !} \exp \left(-\frac{r^{2}}{2 \sigma^{2}}\right), \quad r \geq 0 .
$$


Assuming that $\mu$ is a positive integer and substituting (60), (61), and (63) into (59), we obtain the integral in the form of

$$
\begin{aligned}
\Theta(\sigma, b)= & \int_{0}^{\infty} Q_{\mu}(r, b) p_{R}(r) d r \\
= & \sum_{i=0}^{\infty} \frac{1}{i !(\mu+i-1) !(p-1) ! 2^{2 i+\mu+p-2} \sigma^{2 p}} \\
& \cdot \int_{0}^{\infty} r^{2(i+p)-1} \exp \left(-\frac{\left(\sigma^{2}+1\right) r^{2}}{2 \sigma^{2}}\right) d r \\
& \cdot \int_{b}^{\infty} x^{2(i+\mu)-1} \exp \left(-\frac{x^{2}}{2}\right) d x .
\end{aligned}
$$

Upon evaluating the integrals of (64), we finally simplify the integral of (59) to

$$
\begin{aligned}
\Theta(\sigma, b)= & \sum_{i=0}^{\infty} \sum_{j=0}^{i+\mu-1} \frac{\left(b^{2}\right)^{i+\mu-j-1}}{(i+\mu-j-1) ! 2^{i+\mu-j-1}} \\
& \times\left(\begin{array}{c}
i+p-1 \\
i
\end{array}\right) \frac{\exp \left(-b^{2} / 2\right)}{\sigma^{2 p}\left(1+1 / \sigma^{2}\right)^{i+p}} .
\end{aligned}
$$

\section{ACKNOWLEDGMENT}

The constructive critique of the anonymous reviewers is gratefully acknowledged.

\section{REFERENCES}

[1] R. E. Ziemer and R. L. Peterson, Digital Communications and Spread Spectrum Systems. New York: Macmillan, 1985.

[2] A. Polydoros and C. L. Weber, "A unified approach to serial search spread-spectrum code acquisition-Part I: General theory," IEEE Trans. Commun., vol. COM-32, pp. 542-549, May 1984.

[3] D. V. Sarwate, "Acquisition of direct-sequence spread-spectrum signals," in Wireless Communication-TDMA versus CDMA, S. G. Glisic and P. A. Leppanen, Eds. Norwell, MA: Kluwer, 1997, pp. 121-145.

[4] A. Polydoros and M. K. Simon, "Generalized serial search code acquisition-The equivalent circular state diagram approach," IEEE Trans. Commun., vol. COM-32, pp. 1260-1268, Dec. 1984

[5] J. K. Holmes and C. C. Chen, "Acquisition time performance of PN spread-spectrum," IEEE Trans. Commun., vol. COM-25, pp. 778-783, Aug. 1977.

[6] W. R. Braun, "Performance analysis for the expanding search PN acquisition algorithm," IEEE Trans. Commun., vol. COM-30, pp. 424-435, Mar. 1982.

[7] Y. T. Su, "Rapid code acquisition algorithms employing PN matched filters," IEEE Trans. Commun., vol. 36, pp. 724-733, May 1988.

[8] B. B. Ibrahim and A. H. Aghvami, "Direct sequence spread spectrum matched filter acquisition in frequency-selective Rayleigh fading channel," IEEE J. Select. Areas Commun., vol. 12, pp. 885-890, June 1994.

[9] H. Moon and S. Kim, "Performance of double dwell acquisition with continuous integration detector in DS-CDMA system," in Proc. IEEE VTC'98, Ottawa, ON, Canada, 1998, pp. 2507-2511.

[10] A. Polydoros and C. L. Weber, "A unified approach to serial search spread-spectrum code acquisition-Part II: A matched-filter receiver," IEEE Trans. Commun., vol. COM-32, pp. 550-560, May 1984
[11] U. Cheng, "Performance of a class of parallel spread-spectrum code acquisition schemes in the presence of data modulation," IEEE Trans. Commun., vol. 36, pp. 596-604, May 1988.

[12] E. S. Sourour and S. C. Gupta, "Direct-sequence spread-spectrum parallel acquisition in a fading mobile channel," IEEE Trans. Commun., vol. 38, pp. 992-998, July 1990.

[13] — - "Direct-sequence spread-spectrum parallel acquisition in nonselective and frequency-selective Rician fading channel," IEEE J. Select. Areas Commun., vol. 10, pp. 535-544, Apr. 1992.

[14] R. R. Rick and L. B. Milstein, "Parallel acquisition in mobile DS-CDMA systems," IEEE Trans. Commun., vol. 45, pp. 1466-1476, Nov. 1997.

[15] L. L. Yang and J. Simsa, "Performance evaluation of spread-spectrum code acquisition system using four-state Markov process," in Proc. IEEE Symp. ISSSTA'98, Sun City, South Africa, Sept. 1998, pp. 848-852.

[16] L. L. Yang and L. Hanzo, "Serial acquisition techniques for DS-CDMA signals in frequency-selective multi-user mobile channels," in Proc. IEEE VTC'99, May 1999, pp. 2398-2402.

[17] A. J. Viterbi, CDMA: Principles of Spread Spectrum Communication. Reading, MA: Addison-Wesley, 1995

[18] J. G. Proakis, Digital Communications, 3rd ed. New York: McGrawHill, 1995.

[19] V. M. Jovanovic and E. S. Sousa, "Analysis of noncoherent correlation in DS/BPSK spread spectrum acquisition," IEEE Trans. Commun., vol. 43, no. 2/3/4, pp. 565-573, Feb./Mar./Apr. 1995.

[20] L. B. Milstein, T. S. Rappaport, and R. Barghouti, "Performance evaluation for cellular CDMA," IEEE J. Select. Areas Commun., vol. 10, pp. 680-689, May 1992.

Lie-Liang Yang (M'98) received the B.Eng. degree in communication engineering from Shanghai TieDao University, Shanghai, China, in 1988 and the M.S. and Ph.D. degrees in communications and electronics from Northern Jiaotong University, Beijing, China, in 1991 and 1997, respectively.

From 1991 to 1993, he was a Lecturer with the Department of Electrical Engineering, East-China Jiaotong University. From 1993 to 1997, he was with the Modern Communications Research Institute, Northern Jiaotong University. From June 1997 to December 1997, he was a Visiting Scientist of the Institute of Radio Engineering and Electronics, Academy of Sciences of the Czech Republic. Since December 1997, he has been with the Communication Group, Department of Electronics and Computer Science, University of Southampton, U.K. He has been involved in researching various error-correction coding, modulation schemes, synchronization, and CDMA systems for future generations of wireless mobile communication systems. He has published about 50 papers in journals and conference proceedings.

Dr. Yang received the Royal Society Sino-British Fellowship in 1997.

Lajos Hanzo (M'91-SM'92) received the Masters degree (Dipl. Ing.) in electronics and the Ph.D. degree, both from the Technical University of Budapest, Hungary, in 1976 and 1983, respectively.

During his 24-year career in telecommunications, he has held various research and academic posts in Hungary, Germany, and the United Kingdom. Since 1986, he has been with the Department of Electronics and Computer Science, University of Southampton, U.K., and has been a Consultant to Multiple Access Communications Ltd., U.K. Currently, he holds the Chair in telecommunications. He has coauthored five books on mobile radio communications, published in excess of 300 research papers, organized and chaired conference sessions, presented overview lectures, and was awarded a number of distinctions. Currently, he is managing a research team working on a range of research projects in the field of wireless multimedia communications under the auspices of the Engineering and Physical Sciences Research Council, U.K., the European IST Programme, and the Mobile Virtual Centre of Excellence. He also provides a range of industrial training courses. 